\title{
Electron Microscopy and Electron Energy-Loss Spectroscopy (EELS) of Few-Layer Chemically-Exfoliated Phosphorene Flakes
}

\author{
Hesham M. ElSherif $^{1}$ and Nabil D. Bassim ${ }^{1}$ \\ ${ }^{1 .}$ McMaster University, Materials Science and Engineering Department, Hamilton, ON, Canada.
}

Phosphorene, a 2-dimensional (2-D) form of Black Phosphorous (BP), has superior electronic and mechanical properties that make it a competitor to graphene and transition metal dichalcogenide monolayers (TMDC) materials [1]. It has a direct band gap that is tuneable from 0.3 to $2 \mathrm{eV}$ (near to midIR), by controlling its number of layers, applying an external electric field, changing the contact metal or doping. This novel behaviour makes phosphorene applicable for electronic devices such as light emitting diodes (LED) and solar cells [2]. Like graphene, BP can be exfoliated chemically by ionic liquids using intercalation chemistry into a form of few-layer phosphorene flakes. Recently, we applied the chemical exfoliation process with Chloride Choline Urea (CCU) [3], with an aim to achieve large-size phosphorene flakes with few-layer thickness and to study the chemical bonding as a function of thickness. In this study, a mixture of deep eutectic ionic liquid chloride choline (CC) and urea (U) is prepared at its eutectic point $(\mathrm{U}: \mathrm{CC}=2$ ) for chemically exfoliating $20 \mathrm{mg}$ of bulk BP powder grinded in a nitrogen glove box using a mortar and pestle [3]. The resultant CCU-BP solution was diluted in ethanol to decrease its viscosity and sonicated for 2 hours. Drops of the solution were cast on quantifoil copper for Scanning Transmission Electron Microscopy (STEM). We characterized the exfoliated BP flakes using aberration-corrected STEM-high angle annular dark field (STEM-HAADF) imaging, and electron energy loss spectroscopy (EELS) mapping.

Results of this study had two main observations. First, we were able to link between electron energy-loss near-edge structure (ELNES) of L-edges of phosphorus with the flake thickness. Figure 1 shows two thickness measurements in the zero-loss region with the corresponding ELNES spectrum at the same position. In general, it appears that the flake thickness is directly related to the integration of the fine structure curve (area under the curve) between $\mathrm{P}-\mathrm{L}_{1}$ and $\mathrm{P}-\mathrm{L}_{2,3}$ edges. Therefore, thick flakes present higher signal intensity/integration of P-L edges compared to a thin flake.

Second, we think that we were able to trace the intercalated ionic liquid between the BP layers by using EELS maps and ELNES spectrums. Figure 2 illustrates typical EELS mapping of a region contains both thin and thick BP flakes. For the thick flake located in the upper-right corner, there are rich regions (marked in red arrows) of chlorine, carbon and nitrogen which are the main elements of the ionic liquid used for the exfoliation process. Figure 3 shows three ELNES curves, after subtracting the background, for (1) thin flake, (2) thick flake in a rich CCU region and (3) the same thick flake in a poor CCU region. We found that, although the flake is thick, the CCU-rich regions have lower ELNES relative signal intensities, just like the ELNES of thin BP flakes. Further work is proceeding to investigate the intercalation process by EELS, ELNES, and tomography. This observation would give the opportunity to study both chemistry and mechanisms of ionic liquids intercalation in 2-D materials.

References:

[1] S. Bagheri, N. Mansouri and E. Aghaie, Int. J. Hydrogen Energy 41(7) (2016), p. 4085.

[2] A. Carvalho, M. Wang, X. Zhu, Nat. Rev. Mater. 1(11) (2016), p. 16061.

[3] A. Ng, T. Sutto, B. Matis, Nanotechnology 28(15) (2017), p. 155601. 

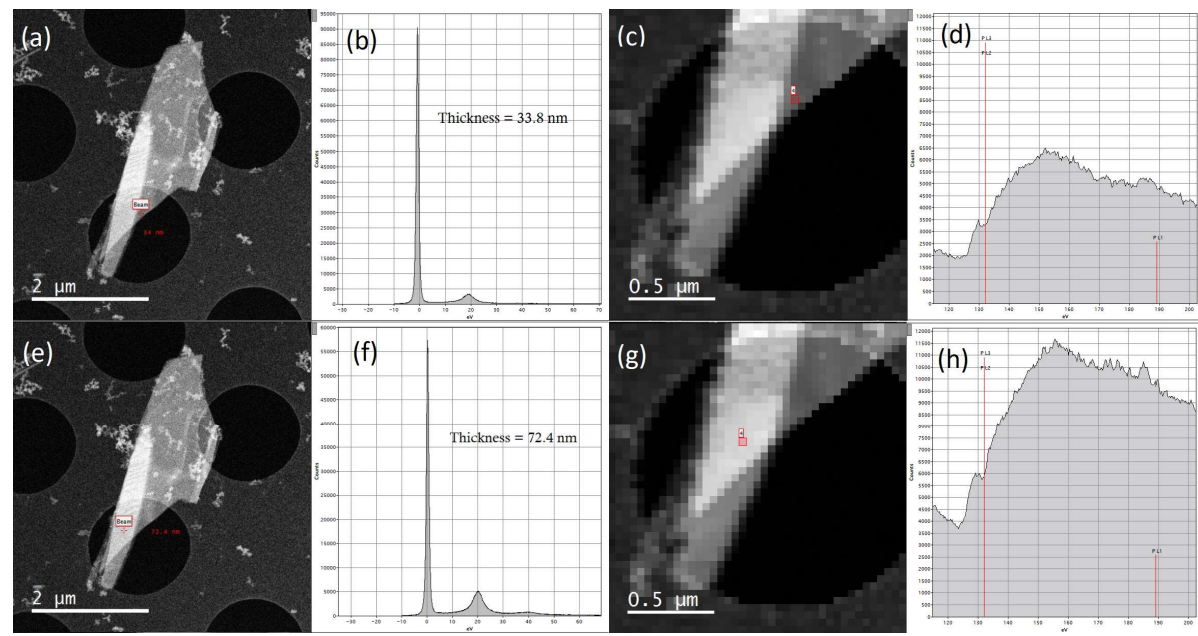

Figure 1. Relation between Phosphorene thickness measured by zero loss EELS in (b) and (f) for 2 positions in a flake as shown in (a) and (e), and the corresponding ELNES in (d) and (h) extracted from the red pixel in (c) and (g), respectively, at the same position where the zero-loss was measured.

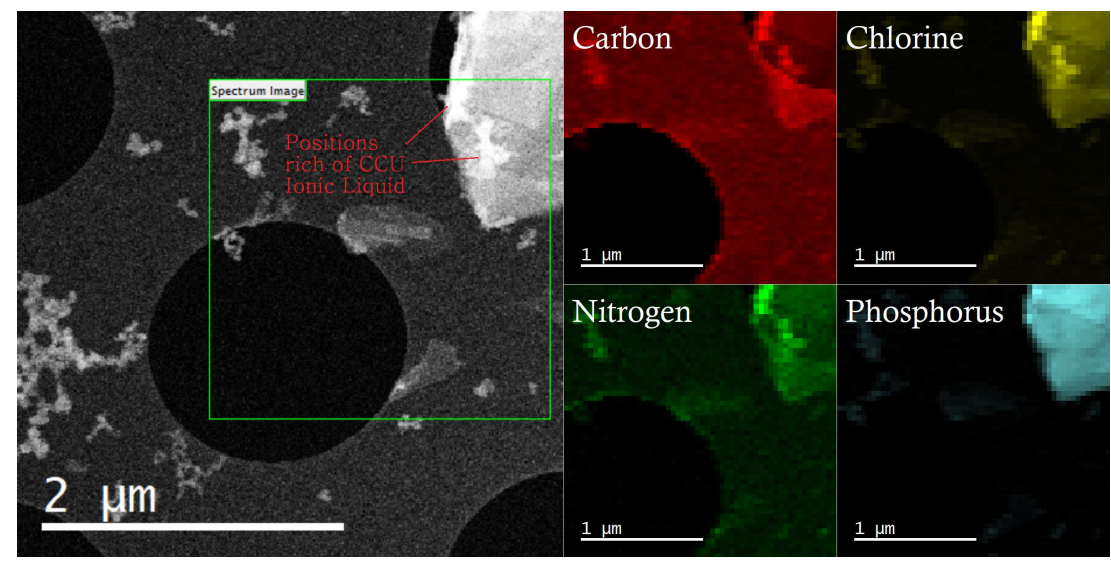

Figure 2. HAADF image (left) and EELS maps (right) of phosphorene flakes. Interesting positions in a thick BP flake are marked in red arrows in up-right corner of the HAADF image. These positions are rich of Nitrogen, chlorine and carbon signals which are the CCU elements.
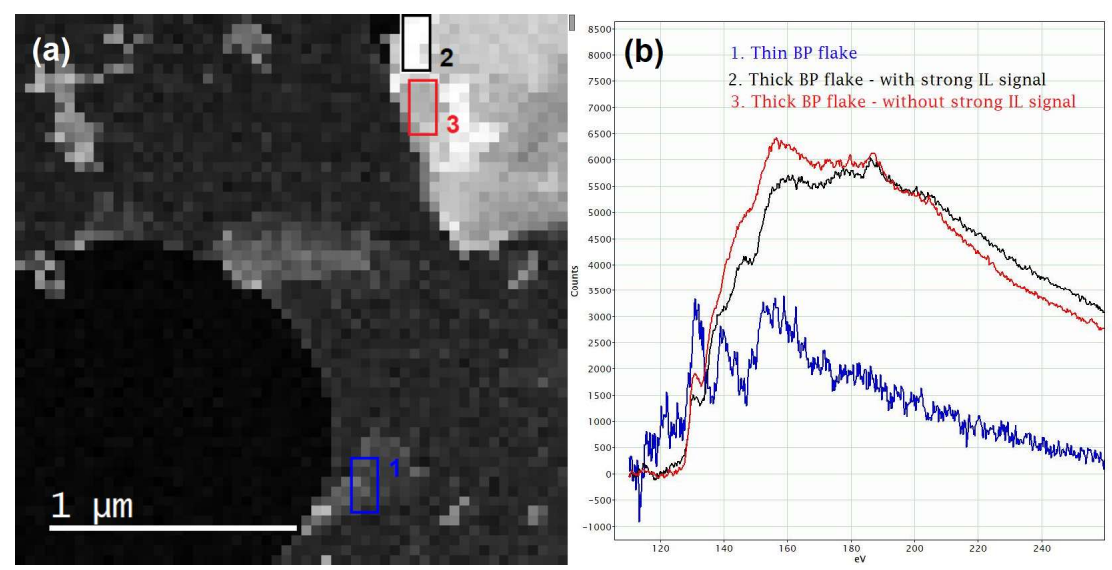

Figure 3. (a) HAADF analog image, (b) subtracted background ELNES within the P-L region for (1) thin BP flake, (2) thick flake at a region with strong ionic liquid signal as shown in figure 2, (3) thick flake in a region with week ionic liquid signal. 